\title{
Main-Chain Calixarene Polymers: Conformational Effects on Polymerization
}

\author{
Yong Yang and Timothy M. Swager* \\ Department of Chemistry and Institute for Soldier Nanotechnologies, Massachusetts Institute of Technology, \\ 77 Massachusetts Avenue, Cambridge, Massachusetts 02139 \\ * Corresponding author. E-mail: tswager@mit.edu
}

\section{Supplemental Information}

\section{Experimental Section}

General Methods and Instrumentation. Nuclear magnetic resonance (NMR) spectra were recorded on Varian Mercury-300 NMR Spectrometer. Chemical shifts are referenced to residual solvent. High-resolution mass spectra were obtained on Bruker Daltonics APEX II 3 Tesla FTICR-MS. Microwave assisted reactions were performed in CEM Discover system (Mattews, NC). HPLC purification was carried out on Agilent 1100 series equipped with a Zorbax Rx-SIL $(9.4 \mathrm{~mm} \mathrm{X} 25 \mathrm{~cm}$ ) column (Agilent Technologies, Inc.). Polymer molecular weights were determined by gel permeation chromatography (GPC) versus polystyrene standards (Polysciences, Warrington, PA) using THF as the eluent at a flow rate of $1.0 \mathrm{ml} / \mathrm{min}$ in a Waters series 1525 HPLC system equipped with four Styragel HT columns operated at $35^{\circ} \mathrm{C}$, Waters in-line degasser AF, 717Plus Autosampler, 2414 refractive index detector, and 2487 dual $\lambda$ absorbance detector. Appropriate amount of sample $(\sim 1 \mathrm{mg} / \mathrm{ml})$ was dissolved in THF and passed through a $0.45 \mu \mathrm{m}$ PTFE syringe filter (Whatman, Clifton, NJ) prior to injection into the column. X-ray diffraction experiments were performed on a Siemens three-circle Platform diffractometer, coupled to a Bruker-APEX CCD detector. All synthetic manipulations were carried out under an argon atmosphere using standard Schlenk techniques or in a nitrogen gas filled 
glovebox (Innovative technologies) unless otherwise noted. Glassware was oven-baked and cooled under $\mathrm{N}_{2}$ atmosphere. All organic extracts were dried over anhydrous $\mathrm{Na}_{2} \mathrm{SO}_{4}$ and filtered prior to solvent removal under reduced pressure. The products were dried under vacuum at least overnight before weighing and calculating yields.

Materials. All solvents were of spectroscopic grade unless otherwise noted. Anhydrous dichloromethane, toluene, acetonitrile, and tetrahydrofuran were obtained using a solvent purification system (Innovative technologies). Anhydrous DMF, diisopropylamine, pyridine, and tetramethylethylenediamine (TMEDA) were purchased from Aldrich as Sure-Seal Bottles and used as received. $p$-tert-Butylcalix[4]arene 2c was prepared by literature methods. ${ }^{1}$ Compounds $\mathbf{2 a}$ and $\mathbf{2 b}$ were synthesized following literature procedures. ${ }^{2}$ All other chemicals were of reagent grade and used as received.

Hex-5-ynyl $\boldsymbol{p}$-toluenesulfonate (1). To a stirred solution of hex-5-yn-1-ol (1.000 g, $0.01 \mathrm{~mol})$ and p-toluenesulfonyl chloride $(2.120 \mathrm{~g}, 0.011 \mathrm{~mol})$ in $20 \mathrm{~mL}$ anhydrous DCM under ice-water bath, was dropwisely added pyridine $(0.97 \mathrm{~mL}, 0.012 \mathrm{~mol})$. The mixture was warmed to room temperature and stirred overnight. The solvent was removed in vacuum and the residue was partitioned between $150 \mathrm{~mL}$ ethyl acetate and $50 \mathrm{~mL} 1 \mathrm{M} \mathrm{HCl}$. The organic extract was washed with $5 \mathrm{wt} \% \mathrm{NaHCO}_{3}$, water, and brine successively. The crude product was purified by flash column chromatography (hexane:ethyl acetate 10:1) to yield a colorless liquid (2.187 g, 87\%). ${ }^{1} \mathrm{H}$ NMR (300 MHz, $\left.\mathrm{CDCl}_{3}\right)$ ): 7.79 (d, 2H, J = 8.1 Hz), 7.35 (d, 2H, J = 8.1 Hz), 4.05 (t, $2 \mathrm{H}, \mathrm{J}=6.3 \mathrm{~Hz}), 2.45(\mathrm{~s}, 3 \mathrm{H}), 2.16(\mathrm{dt}, 2 \mathrm{H}, \mathrm{J}=2.6,6.9 \mathrm{~Hz}), 1.92(\mathrm{t}, 1 \mathrm{H}, \mathrm{J}=2.6 \mathrm{~Hz}), 1.78(\mathrm{~m}, 2 \mathrm{H}), 1.55(\mathrm{~m}, 2 \mathrm{H})$.

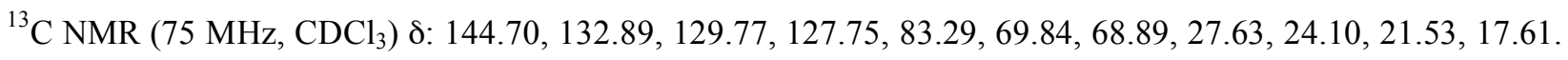
HRMS-ESI (m/z) for $\mathrm{C}_{13} \mathrm{H}_{16} \mathrm{O}_{3} \mathrm{~S}$ calcd. [M+Na] $]^{+}$: 275.0712; found: 275.0703 .

\section{General methods for synthesizing 3a, $4 a, 3 b$, and $4 b$.}

Method A. 2a or $\mathbf{2 b}$, and excess $\mathrm{Cs}_{2} \mathrm{CO}_{3}$ were suspended in dry MeCN. The mixture was heated to reflux for $1 \mathrm{~h}$ and then added with excess $\mathbf{1}$ (for 3a and $\mathbf{3 b}$ ) or propargyl bromide (for $\mathbf{4 a}$ and $\mathbf{4 b}$ ). The mixture was refluxed for $2 \mathrm{~d}$ before cooled to room temperature. The insoluble solid was filtered and solvent was removed under reduced pressure. The residue was partitioned between DCM and $1 \mathrm{M} \mathrm{HCl}$. The organic extract was washed with $1 \mathrm{M} \mathrm{HCl}$, water, and brine. After removing the solvent, the resultant solid was dissolved in minimum amount of DCM and precipitated to large amount of fresh $\mathrm{MeOH}$. The white precipitate was filtered after sitting in $-40{ }^{\circ} \mathrm{C}$ freezer overnight followed by column chromatography. 
Method $\boldsymbol{B}$. NaH was used as the base instead of $\mathrm{Cs}_{2} \mathrm{CO}_{3}$ and THF/DMF (9/1) was used as the solvent instead of $\mathrm{MeCN}$.

\section{5,11,17,23-Tetrakis(1,1-dimethylethyl)-25,27-bis(hex-5-ynyloxy)-26,28-bis(benzyloxy)calix[4]arene} (3a).

Method A. 2a (5.000 g, $6.035 \mathrm{mmol}), \mathrm{Cs}_{2} \mathrm{CO}_{3}(15.746 \mathrm{~g}, 48.280 \mathrm{mmol}), \mathrm{MeCN}(100 \mathrm{~mL})$, and $1(3.807 \mathrm{~g}$, $15.088 \mathrm{mmol})$. The product was obtained as a $67 / 33$ mixture of paco/cone conformers $(5.073 \mathrm{~g}, 85 \%)$ after column chromatography (hexane: ethyl acetate 4:1).

Method B. 2a (1.000 g, $1.207 \mathrm{mmol})$, NaH (0.076 g, $3.018 \mathrm{mmol})$, THF (18 mL), DMF (2 mL), and 1 $(0.761 \mathrm{~g}, 3.018 \mathrm{mmol})$. The product was obtained as a 50/50 mixture of paco/cone conformers $(1.048 \mathrm{~g}, 88 \%)$ after column chromatography (hexane: ethyl acetate 4:1).

Further column chromatography by eluting with hexane/DCM (5/4) afforded 3a-paco, 3a-cone, and a small amount of overlap of the two conformers. Pure 3a-paco and 3a-cone were recrystallized from $\mathrm{CHCl}_{3} / \mathrm{MeOH}$ respectively before polymerizations. For some polymerizations, monomer 3a-paco was additionally purified by semi-preparative HPLC (10 v/v\% ethyl acetate in heptane). 3a-paco: ${ }^{1} \mathrm{H}$ NMR (300

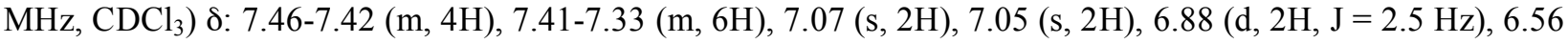
$(\mathrm{d}, 2 \mathrm{H}, \mathrm{J}=2.5 \mathrm{~Hz}), 4.73(\mathrm{~d}, 2 \mathrm{H}, \mathrm{J}=10.7 \mathrm{~Hz}), 4.59(\mathrm{~d}, 2 \mathrm{H}, \mathrm{J}=10.7 \mathrm{~Hz}), 4.08(\mathrm{~d}, 2 \mathrm{H}, \mathrm{J}=12.6 \mathrm{~Hz}), 3.67(\mathrm{~d}, 2 \mathrm{H}$, $\mathrm{J}=12.7 \mathrm{~Hz}), 3.63(\mathrm{t}, 2 \mathrm{H}, \mathrm{J}=7.9 \mathrm{~Hz}), 3.60(\mathrm{~d}, 2 \mathrm{H}, \mathrm{J}=12.7 \mathrm{~Hz}), 3.42(\mathrm{t}, 2 \mathrm{H}, \mathrm{J}=8.5 \mathrm{~Hz}), 2.99(\mathrm{~d}, 2 \mathrm{H}, \mathrm{J}=12.6$ Hz), $2.26(\mathrm{dt}, 2 \mathrm{H}, \mathrm{J}=2.6,7.0 \mathrm{~Hz}), 1.98(\mathrm{t}, 1 \mathrm{H}, \mathrm{J}=2.6 \mathrm{~Hz}), 1.96(\mathrm{~m}, 2 \mathrm{H}), 1.93(\mathrm{t}, 1 \mathrm{H}, \mathrm{J}=2.6 \mathrm{~Hz}), 1.80(\mathrm{dt}, 2 \mathrm{H}$, $\mathrm{J}=2.6,7.6 \mathrm{~Hz}), 1.60(\mathrm{~m}, 2 \mathrm{H}), 1.35(\mathrm{~s}, 9 \mathrm{H}), 1.27(\mathrm{~m}, 2 \mathrm{H}), 1.07(\mathrm{~s}, 9 \mathrm{H}), 1.05(\mathrm{~s}, 18 \mathrm{H}), 0.89(\mathrm{~m}, 2 \mathrm{H}) .{ }^{13} \mathrm{C}$ NMR $\left(75 \mathrm{MHz}, \mathrm{CDCl}_{3}\right) \delta: 155.35,153.72,153.61,145.06,144.01,143.32,138.08,135.92,132.89,132.40,132.09$, $129.62,128.62,128.20,128.11,126.22,125.74,125.60,85.25,84.23,77.04,72.72,72.24,68.98,68.14,37.98$, $34.31,33.99,33.92,31.95,31.72,31.61,31.56,30.23,28.16,25.18,25.15,22.94,18.61,18.41,14.38,32.12$, 31.70. HRMS-ESI (m/z) for $\mathrm{C}_{70} \mathrm{H}_{84} \mathrm{O}_{4}$ calcd. $[\mathrm{M}+\mathrm{H}]^{+}$: 989.6442; found: 989.6447 . 3a-cone: ${ }^{1} \mathrm{H}$ NMR (300 $\left.\mathrm{MHz}, \mathrm{CDCl}_{3}\right)$ d: $7.48(\mathrm{~m}, 4 \mathrm{H}), 7.39(\mathrm{~m}, 6 \mathrm{H}), 7.10(\mathrm{~s}, 4 \mathrm{H}), 6.49(\mathrm{~s}, 4 \mathrm{H}), 4.65(\mathrm{~s}, 4 \mathrm{H}), 4.41(\mathrm{~d}, 4 \mathrm{H}, \mathrm{J}=12.4 \mathrm{~Hz})$, $3.74(\mathrm{t}, 4 \mathrm{H}, \mathrm{J}=8.4 \mathrm{~Hz}), 3.12(\mathrm{~d}, 4 \mathrm{H}, \mathrm{J}=12.4 \mathrm{~Hz}), 1.99(\mathrm{t}, 2 \mathrm{H}, \mathrm{J}=2.6 \mathrm{~Hz}), 1.81(\mathrm{dt}, 4 \mathrm{H}, \mathrm{J}=2.6,7.5 \mathrm{~Hz}), 1.72$

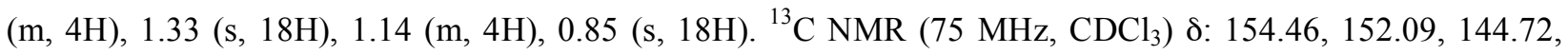
$144.33,137.83,135.45,132.10,129.71,128.30,128.15,125.36,124.51,84.89,78.03,74.16,68.08,34.04$, 33.60, 31.72, 31.14, 29.06, 25.00, 18.38. HRMS-ESI (m/z) for $\mathrm{C}_{70} \mathrm{H}_{84} \mathrm{O}_{4}$ calcd. $[\mathrm{M}+\mathrm{H}]^{+}:$989.6442; found: 
989.6452.

\section{5,11,17,23-Tetrakis(1,1-dimethylethyl)-25,27-bis(prop-2-ynyloxy)-26,28-bis(benzyloxy)calix[4]aren} e (4a).

Method A. 2a (0.800 g, $0.966 \mathrm{mmol}), \mathrm{Cs}_{2} \mathrm{CO}_{3}(2.517 \mathrm{~g}, 7.725 \mathrm{mmol}), \mathrm{MeCN}(50 \mathrm{~mL})$, and propargyl bromide ( $80 \mathrm{wt} \%$ in toluene, $0.86 \mathrm{~mL}, 7.725 \mathrm{mmol})$. The product was obtained as pure paco conformer $(0.805$ g, 92\%) after column chromatography (hexane: ethyl acetate 4:1).

Method B. 2a (0.500 g, $0.6 \mathrm{mmol})$, NaH (0.076 g, $3.0 \mathrm{mmol})$, THF (45 mL), DMF (5 mL), and propargyl bromide ( $80 \mathrm{wt} \%$ in toluene, $0.67 \mathrm{~mL}, 6.0 \mathrm{mmol})$. The product was obtained as a 50/50 mixture of paco/cone conformers (0.455 g, 84\%) after column chromatography (hexane: ethyl acetate 4:1). Pure 4a-cone was obtained after recrystallization from $\mathrm{CHCl}_{3} / \mathrm{MeOH}$ twice.

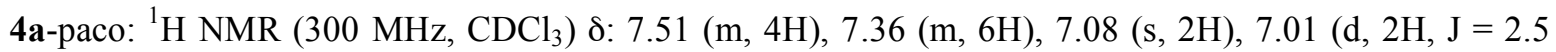
$\mathrm{Hz}), 6.94(\mathrm{~s}, 2 \mathrm{H}), 6.51(\mathrm{~d}, 2 \mathrm{H}, \mathrm{J}=2.5 \mathrm{~Hz}), 4.72(\mathrm{~d}, 2 \mathrm{H}, \mathrm{J}=10.7 \mathrm{~Hz}), 4.66(\mathrm{~d}, 2 \mathrm{H}, \mathrm{J}=10.7 \mathrm{~Hz}), 4.41(\mathrm{~d}, 2 \mathrm{H}, \mathrm{J}=$ $2.5 \mathrm{~Hz}), 4.28(\mathrm{~d}, 2 \mathrm{H}, \mathrm{J}=13.1 \mathrm{~Hz}), 4.15(\mathrm{~d}, 2 \mathrm{H}, \mathrm{J}=2.5 \mathrm{~Hz}), 3.69(\mathrm{~d}, 2 \mathrm{H}, \mathrm{J}=13.5 \mathrm{~Hz}), 3.63(\mathrm{~d}, 2 \mathrm{H}, \mathrm{J}=13.5 \mathrm{~Hz})$, $3.08(\mathrm{~d}, 2 \mathrm{H}, \mathrm{J}=13.1 \mathrm{~Hz}), 2.47(\mathrm{t}, 1 \mathrm{H}, \mathrm{J}=2.5 \mathrm{~Hz}), 2.16(\mathrm{t}, 1 \mathrm{H}, \mathrm{J}=2.5 \mathrm{~Hz}), 1.35(\mathrm{~s}, 9 \mathrm{H}), 1.06(\mathrm{~s}, 18 \mathrm{H}), 0.87(\mathrm{~s}$,

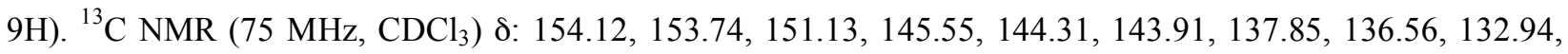
$131.97,131.32,129.11,128.47,128.33,127.91,125.99,125.33,125.27,81.19,80.96,76.83,74.51,74.33$, 59.02, 57.54, 37.48, 34.12, 33.73, 33.48, 32.10, 31.64, 31.43, 31.00. HRMS-ESI (m/z) for $\mathrm{C}_{64} \mathrm{H}_{72} \mathrm{O}_{4}$ calcd. $[\mathrm{M}+\mathrm{Na}]^{+}:$927.5323; found: 927.5343. 4a-cone: ${ }^{1} \mathrm{H} \mathrm{NMR}\left(300 \mathrm{MHz}, \mathrm{CDCl}_{3}\right)$ 8: $7.53(\mathrm{~m}, 4 \mathrm{H}), 7.37(\mathrm{~m}, 6 \mathrm{H})$, 7.09 (s, 4H), $6.44(\mathrm{~s}, 4 \mathrm{H}), 4.77(\mathrm{~s}, 4 \mathrm{H}), 4.74(\mathrm{~d}, 4 \mathrm{H}, \mathrm{J}=2.5 \mathrm{~Hz}), 4.48(\mathrm{~d}, 4 \mathrm{H}, \mathrm{J}=12.8 \mathrm{~Hz}), 3.08(\mathrm{~d}, 4 \mathrm{H}, \mathrm{J}=12.8$

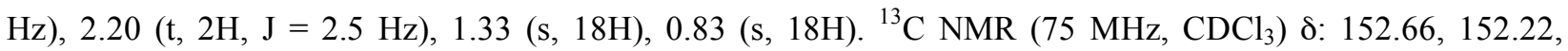
$145.74,144.47,138.11,136.41,131.93,129.06,128.24,127.77,125.33,124.40,81.40,77.74,74.39,59.37$, 34.12, 33.57, 31.89, 31.65, 31.43, 31.08. HRMS-ESI (m/z) for $\mathrm{C}_{64} \mathrm{H}_{72} \mathrm{O}_{4}$ calcd. $[\mathrm{M}+\mathrm{Na}]^{+}:$927.5323; found: 927.5319

\section{5,11,17,23-Tetrakis(1,1-dimethylethyl)-25,27-bis(hex-5-ynyloxy)-26,28-bis(methoxy)calix[4]arene} $(3 \mathbf{b})$.

Method A. $2 \mathrm{~b}$ (2.500 g, $3.698 \mathrm{mmol}), \mathrm{Cs}_{2} \mathrm{CO}_{3}(9.649 \mathrm{~g}, 29.586 \mathrm{mmol}), \mathrm{MeCN}(100 \mathrm{~mL})$, and 1 (2.332 g, $9.245 \mathrm{mmol})$. The product was obtained as a white solid (2.162 g, 70\%) after column chromatography (hexane: ethyl acetate 4:1). ${ }^{1} \mathrm{H}$ NMR (300 MHz, $\left.\mathrm{CDCl}_{3}\right)$ 8: 7.25-6.42 (bm, 8H), 4.34-3.06 (bm, 18H), 2.37-1.55 (bm, 
10H), 1.40-1.25 (bm, 18H), 1.04 (bs, 9H), 0.80 (bs, 4H). ${ }^{3}$ HRMS-ESI (m/z) for $\mathrm{C}_{58} \mathrm{H}_{76} \mathrm{O}_{4}$ calcd. [M+Na] 859.5636; found: 859.5650 .

\section{5,11,17,23-Tetrakis(1,1-dimethylethyl)-25,27-bis(prop-2-ynyloxy)-26,28-bis(methoxy)calix[4]arene} (4b).

Method B. 2b (1.010 g, $1.5 \mathrm{mmol})$, NaH (0.190 g, $7.5 \mathrm{mmol})$, THF (45 mL), DMF (5 mL), and propargyl bromide ( $80 \mathrm{wt} \%$ in toluene, $1.67 \mathrm{~mL}, 15.0 \mathrm{mmol})$. The product was obtained as a white solid $(0.740 \mathrm{~g}, 66 \%)$ after column chromatography (hexane: ethyl acetate 4:1). ${ }^{1} \mathrm{H}$ NMR $\left(300 \mathrm{MHz}, \mathrm{CDCl}_{3}\right)$ 8: 7.37-6.58 (bm, 8H), 4.58-4.32 (bm, 4H), $4.14(\mathrm{bd}, 2 \mathrm{H}, \mathrm{J}=13.2 \mathrm{~Hz}), 3.97(\mathrm{bs}, 2 \mathrm{H}), 3.82(\mathrm{bd}, 2 \mathrm{H}, \mathrm{J}=13.2 \mathrm{~Hz}), 3.70(\mathrm{bd}, 2 \mathrm{H}, \mathrm{J}=$ $13.2 \mathrm{~Hz}$ ), 3.32-3.14 (bm, 6H), 2.47 (bs, $2 \mathrm{H}), 1.44-0.92$ (bm, 36H). ${ }^{4}$ HRMS-ESI (m/z) for $\mathrm{C}_{52} \mathrm{H}_{64} \mathrm{O}_{4}$ calcd. $[\mathrm{M}+\mathrm{Na}]^{+}:$775.4697; found: 775.4682 .

\section{General method for synthesizing $3 c$ and $4 c$.}

2c and 1.1 equiv. $\mathrm{K}_{2} \mathrm{CO}_{3}$ were suspended in dry $\mathrm{MeCN}$. The mixture was heated to reflux for $1 \mathrm{~h}$ and then added dropwisely with 2 equiv. 1 (for $\mathbf{3 c}$ ) or propargyl bromide (for $\mathbf{4 c}$ ). The mixture was refluxed for $2 \mathrm{~d}$ before cooled to room temperature. The insoluble solid was filtered and solvent was removed under reduced pressure. The residue was partitioned between DCM and $1 \mathrm{M} \mathrm{HCl}$. The organic extract was washed with $1 \mathrm{M}$ $\mathrm{HCl}$, water, and brine. After removing the solvent, the resultant solid was dissolved in minimum amount of DCM and precipitated to large amount of fresh $\mathrm{MeOH}$. The white precipitate was filtered after sitting in -40 ${ }^{\circ} \mathrm{C}$ freezer overnight followed by column chromatography.

5,11,17,23-Tetrakis(1,1-dimethylethyl)-25,27-bis(hex-5-ynyloxy)-26,28-dihydroxycalix[4]arene (3c). $\mathbf{2 c}$ (with one toluene adduct) (1.000 g, $1.35 \mathrm{mmol}), \mathrm{K}_{2} \mathrm{CO}_{3}(0.206 \mathrm{~g}, 1.49 \mathrm{mmol}), \mathrm{MeCN}(30 \mathrm{~mL})$, and $\mathbf{1}(0.682$ $\mathrm{g}, 2.70 \mathrm{mmol})$. The product was obtained as a white solid $(0.827 \mathrm{~g}, 76 \%)$ after column chromatography (hexane: ethyl acetate 4:1). ${ }^{1} \mathrm{H}$ NMR (300 MHz, $\left.\mathrm{CDCl}_{3}\right)$ d: 7.68 (s, 2H), 7.04 (s, 4H), 6.83 (s, 4H), 4.26 (d, 4H, J = 12.9 Hz), 4.00 (t, 4H, J = 6.2 Hz), 3.31 (d, 4H, J = 12.9 Hz), $2.40(\mathrm{dt}, 4 \mathrm{H}, \mathrm{J}=2.6,7.0 \mathrm{~Hz}), 2.13(\mathrm{~m}, 4 \mathrm{H}), 2.00$ (t, 2H,

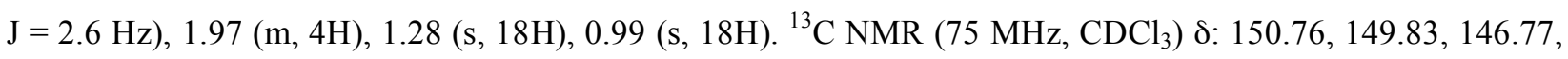
$141.31,132.64,127.65,125.46,125.03,84.20,75.76,68.78,33.94,33.78,31.77,31.68,31.03,29.04,25.01$, 18.27. HRMS-ESI (m/z) for $\mathrm{C}_{56} \mathrm{H}_{72} \mathrm{O}_{4}$ calcd. $[\mathrm{M}+\mathrm{Na}]^{+}:$: 831.5323; found: 831.5301 .

\section{5,11,17,23-Tetrakis(1,1-dimethylethyl)-25,27-bis(prop-2-ynyloxy)-26,28-dihydroxycalix[4]arene}

(4c). 2c (with one toluene adduct) $(2.000 \mathrm{~g}, 2.70 \mathrm{mmol}), \mathrm{K}_{2} \mathrm{CO}_{3}(0.410 \mathrm{~g}, 2.98 \mathrm{mmol}), \mathrm{MeCN}(50 \mathrm{~mL})$, and 
propargyl bromide ( $80 \mathrm{wt} \%$ in toluene, $0.60 \mathrm{~mL}, 5.40 \mathrm{mmol})$. The product was obtained as a white solid (1.342

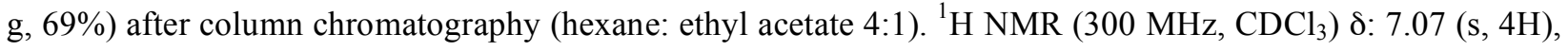
$6.73(\mathrm{~s}, 4 \mathrm{H}), 6.51(\mathrm{~s}, 2 \mathrm{H}), 4.75(\mathrm{~d}, 4 \mathrm{H}, \mathrm{J}=2.4 \mathrm{~Hz}), 4.38(\mathrm{~d}, 4 \mathrm{H}, \mathrm{J}=13.3 \mathrm{~Hz}), 3.34(\mathrm{~d}, 4 \mathrm{H}, \mathrm{J}=13.3 \mathrm{~Hz}), 2.54(\mathrm{t}$,

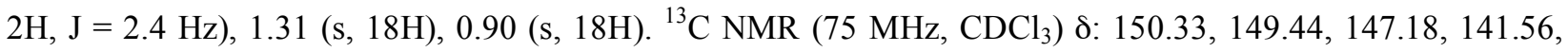
$132.53,127.97,125.49,124.98,78.75,76.29,63.25,33.85,33.82,32.00,31.69,30.92$. HRMS-ESI (m/z) for $\mathrm{C}_{50} \mathrm{H}_{60} \mathrm{O}_{4}$ calcd. $[\mathrm{M}+\mathrm{Na}]^{+}:$747.4384; found: 747.4367 .

\section{General method for polymerizations.}

To a $10 \mathrm{~mL}$ microwave reactor compatible tube, were added $0.1 \mathrm{mmol}$ monomer, $0.125 \mathrm{mmol}$ benzoquinone, $0.032 \mathrm{mmol} \mathrm{CuI}, 0.0025 \mathrm{mmol} \mathrm{Pd}\left(\mathrm{PPh}_{3}\right)_{2} \mathrm{Cl}_{2}, 0.4 \mathrm{~mL}$ toluene, and $0.15 \mathrm{~mL}{ }^{\mathrm{i}} \mathrm{Pr}_{2} \mathrm{NH}$ or TMEDA. After the mixture was heated in microwave reactor at $65{ }^{\circ} \mathrm{C}$ for $1.5 \mathrm{~h}$, it was cooled to room temperature followed by diluting with $75 \mathrm{~mL} \mathrm{CHCl}$. The organic layer was washed successively with $1 \mathrm{M} \mathrm{HCl}$, saturated $\mathrm{NaHCO}_{3}$ aqueous solution, $30 \mathrm{wt} \% \mathrm{NH}_{4} \mathrm{OH}$ aqueous solution, $1 \mathrm{M} \mathrm{HCl}$, water, and brine before concentrated to viscous liquid. The residue was precipitated to large amount of $\mathrm{MeOH}$. After sitting in $-40{ }^{\circ} \mathrm{C}$ freezer overnight, the precipitate was filtered and washed with several portions of fresh $\mathrm{MeOH}$. Polymers were off-white to light pale brown flakes and oligomers were powders with the same color.

Poly(3a-paco). Some polymerizations of 3a-paco were performed under $65{ }^{\circ} \mathrm{C}$ oil bath in order to monitor the gelation. Polymerization of 3a-paco before HPLC gelled in $10 \mathrm{~min}$ and polymerization of 3a-paco after HPLC gelled overnight in $65{ }^{\circ} \mathrm{C}$ oil bath. The gels completely dissolved into $\mathrm{CHCl}_{3}$ after sonication. ${ }^{1} \mathrm{H}$ NMR (300 MHz, $\left.\mathrm{CDCl}_{3}\right)$ ઈ: 7.46-7.31 (m, 10H), 7.07 (s, 2H), 7.05 (s, 2H), 6.86 (bs, 2H), 6.56 (bs, 2H), $4.72(\mathrm{~d}$, $2 \mathrm{H}, \mathrm{J}=10.5 \mathrm{~Hz}), 4.56(\mathrm{~d}, 2 \mathrm{H}, \mathrm{J}=10.5 \mathrm{~Hz}), 4.06(\mathrm{~d}, 2 \mathrm{H}, \mathrm{J}=12.1 \mathrm{~Hz}), 3.62(\mathrm{bm}, 6 \mathrm{H}), 3.35(\mathrm{t}, 2 \mathrm{H}, \mathrm{J}=8.5 \mathrm{~Hz})$, $2.99(\mathrm{~d}, 2 \mathrm{H}, \mathrm{J}=12.1 \mathrm{~Hz}), 2.30(\mathrm{t}, 2 \mathrm{H}, \mathrm{J}=6.3 \mathrm{~Hz}), 1.90(\mathrm{~m}, 2 \mathrm{H}), 1.79(\mathrm{t}, 2 \mathrm{H}, \mathrm{J}=7.4 \mathrm{~Hz}), 1.59(\mathrm{~m}, 2 \mathrm{H}), 1.34(\mathrm{~s}$, 9H), $1.22(\mathrm{~m}, 2 \mathrm{H}), 1.07(\mathrm{~s}, 9 \mathrm{H}), 1.06(\mathrm{~s}, 9 \mathrm{H}), 1.05(\mathrm{~s}, 9 \mathrm{H}), 0.88(\mathrm{~m}, 2 \mathrm{H})$.

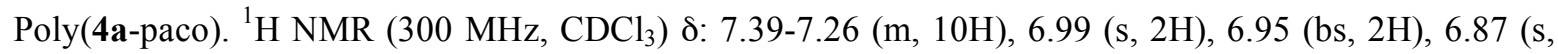
2H), $6.44(\mathrm{bs}, 2 \mathrm{H}), 4.64(\mathrm{~d}, 2 \mathrm{H}, \mathrm{J}=10.5 \mathrm{~Hz}), 4.54(\mathrm{~d}, 2 \mathrm{H}, \mathrm{J}=10.5 \mathrm{~Hz}), 4.47(\mathrm{~s}, 2 \mathrm{H}), 4.18(\mathrm{~d}, 2 \mathrm{H}, \mathrm{J}=12.8 \mathrm{~Hz})$, $4.07(\mathrm{~s}, 2 \mathrm{H}), 3.64(\mathrm{~d}, 2 \mathrm{H}, \mathrm{J}=13.9 \mathrm{~Hz}), 3.56(\mathrm{~d}, 2 \mathrm{H}, \mathrm{J}=13.9 \mathrm{~Hz}), 2.98(\mathrm{~d}, 2 \mathrm{H}, \mathrm{J}=12.8 \mathrm{~Hz}), 1.27(\mathrm{~s}, 9 \mathrm{H}), 1.04$ (s, 18H), $0.82(\mathrm{~s}, 9 \mathrm{H})$.

Reduction of poly(3a-paco). A solution of $20 \mathrm{mg}$ poly(3a-paco) in $5 \mathrm{~mL}$ toluene was heated to reflux followed by addition of $p$-toluenesulfonhydrazide $(77 \mathrm{mg}, 0.4 \mathrm{mmol})$ and tripropylamine $(77 \mu \mathrm{L}, 0.4 \mathrm{mmol})$. 
After refluxing for $1.5 \mathrm{~h}$, another portion of $p$-toluenesulfonhydrazide and tripropylamine with the same amount was added. The mixture was refluxed for additional $2 \mathrm{~h}$ followed by passing through an alumina plug when it was hot. After washing with excess $\mathrm{CHCl}_{3}$, the combined organics was washed with brine twice and concentrated to thick oily liquid. The residue was precipitated to large amount of $\mathrm{MeOH}$. The precipitate was filtered and washed with excess fresh $\mathrm{MeOH}$. The reduced polymer was obtained as an off-white flake solid (19 mg, 95\%). $\mathrm{M}_{\mathrm{n}}=83 \mathrm{kD},{ }^{5} \mathrm{PDI}=2.16 .{ }^{1} \mathrm{H}$ NMR (300 MHz, $\left.\mathrm{CDCl}_{3}\right)$ 8: 7.45-7.33 (m, 10H), 7.07 (s, 2H), $7.03(\mathrm{~s}, 2 \mathrm{H}), 6.88(\mathrm{bs}, 2 \mathrm{H}), 6.55(\mathrm{bs}, 2 \mathrm{H}), 4.72(\mathrm{~d}, 2 \mathrm{H}, \mathrm{J}=10.8 \mathrm{~Hz}), 4.61(\mathrm{~d}, 2 \mathrm{H}, \mathrm{J}=10.8 \mathrm{~Hz}), 4.11(\mathrm{~d}, 2 \mathrm{H}, \mathrm{J}=$ $12.5 \mathrm{~Hz}), 3.63(\mathrm{bm}, 6 \mathrm{H}), 3.45(\mathrm{~m}, 2 \mathrm{H}), 2.98(\mathrm{~d}, 2 \mathrm{H}, \mathrm{J}=12.5 \mathrm{~Hz}), 1.86(\mathrm{~m}, 2 \mathrm{H}), 1.55(\mathrm{~m}, 2 \mathrm{H}), 1.35(\mathrm{~s}, 9 \mathrm{H})$, 1.25-1.15 (bm, 8H), 0.95-0.68 (bm, 8H), $1.06(\mathrm{~s}, 18 \mathrm{H}), 1.05(\mathrm{~s}, 9 \mathrm{H})$.

Deprotection of poly(3a-paco). To a solution of $10 \mathrm{mg}$ poly(3a-paco) in $1 \mathrm{~mL} \mathrm{CHCl}_{3}$, was added $\operatorname{TMSBr}(27 \mu \mathrm{L}, 0.2 \mathrm{mmol})$. The mixture was heated at $40^{\circ} \mathrm{C}$ for $2 \mathrm{~h}$ before cooled to room temperature. A small amount of methanol-water was added to quench the reaction. After stirring for $0.5 \mathrm{~h}$, the solvent was removed and the residue was taken up in $50 \mathrm{~mL} \mathrm{CHCl}$. The organics was washed with $30 \mathrm{wt} \% \mathrm{Na}_{2} \mathrm{~S}_{2} \mathrm{O}_{3}$ and brine and then concentrated to viscous oil. The residue was triturated with hexane and the precipitate was filtered. The deprotected polymer was obtained as an off-white flake solid (7 mg, 86\%). $\mathrm{M}_{\mathrm{n}}=42 \mathrm{kD},{ }^{6} \mathrm{PDI}=2.04 .{ }^{1} \mathrm{H}$ NMR

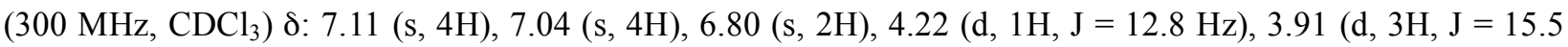
$\mathrm{Hz}), 3.71(\mathrm{~d}, 3 \mathrm{H}, \mathrm{J}=15.5 \mathrm{~Hz}), 3.55(\mathrm{bs}, 4 \mathrm{H}), 3.28(\mathrm{~d}, 1 \mathrm{H}, \mathrm{J}=12.8 \mathrm{~Hz}), 2.42(\mathrm{t}, 2 \mathrm{H}, \mathrm{J}=5.0 \mathrm{~Hz}), 2.05(\mathrm{~m}, 2 \mathrm{H})$, $1.94(\mathrm{~m}, 2 \mathrm{H}), 1.71(\mathrm{t}, 2 \mathrm{H}, \mathrm{J}=6.7 \mathrm{~Hz}), 1.61(\mathrm{~m}, 2 \mathrm{H}), 1.29-1.25(\mathrm{~m}, 36 \mathrm{H}), 1.06(\mathrm{~m}, 2 \mathrm{H})$. 
Table S1. Base and solvent effects on paco/cone ratios of $\mathbf{3 a}$ and $\mathbf{4 a}$.

\begin{tabular}{ccccc}
\hline entry & compound & solvent & base & paco:cone ${ }^{\mathrm{a}}$ \\
\hline 1 & $\mathbf{3 a}$ & THF:DMF $(9: 1)$ & $\mathrm{NaH}$ & $50: 50$ \\
2 & $\mathbf{3 a}$ & MeCN & $\mathrm{Cs}_{2} \mathrm{CO}_{3}$ & $67: 33$ \\
3 & $\mathbf{4 a}$ & THF:DMF $(9: 1)$ & $\mathrm{NaH}$ & $50: 50$ \\
4 & $\mathbf{4 a}$ & MeCN & $\mathrm{Cs}_{2} \mathrm{CO}_{3}$ & $>100: 1$ \\
\hline${ }^{a}$ Determined by ${ }^{1} \mathrm{H}$ NMR spectroscopy. & &
\end{tabular}

Scheme S1. Selected Pd-free acetylenic coupling polymerizations.

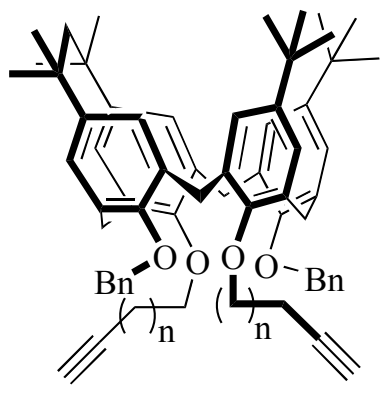

(a) $\mathrm{n}=0$, cone, dimer, $15 \%$

$n=3$, cone, dimer and trimer, $45 \%$ (a) $\mathrm{Cu}(\mathrm{OAc})_{2}, \mathrm{CuI}$, pyridine, air, $60^{\circ} \mathrm{C}$

(b) $\mathrm{CuCl}$, TMEDA, pyridine, o-DCB, $\mathrm{O}_{2}, 75^{\circ} \mathrm{C}$

(b) $\mathrm{n}=0$, cone, $\mathrm{Mn}=4 \mathrm{kD}, 85 \%$

$\mathrm{n}=3$, cone, $\mathrm{Mn}=8 \mathrm{kD}, 95 \%$

Table S2. Selected Sonogashira coupling polymerizations.
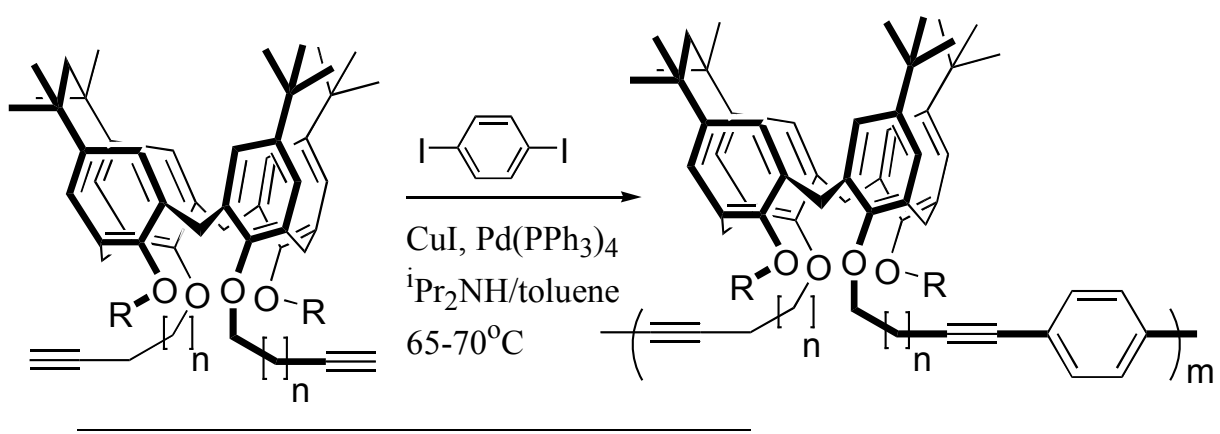

\begin{tabular}{ccccccc}
\hline entry & monomer & $\mathrm{R}$ & $\mathrm{n}$ & $\begin{array}{c}\mathrm{M}_{\mathrm{n}} \\
(\mathrm{kD})\end{array}$ & PDI & $\begin{array}{c}\text { yield } \\
(\%)\end{array}$ \\
\hline 1 & 3a-cone & $\mathrm{Bn}$ & 3 & 16 & 2.11 & quant. \\
2 & 3c & $\mathrm{H}$ & 3 & 16 & 1.76 & quant. \\
3 & 4a-cone & $\mathrm{Bn}$ & 0 & 11 & 1.94 & 89 \\
4 & 4a-paco & $\mathrm{Bn}$ & 0 & 11 & 1.39 & 67 \\
\hline
\end{tabular}

Figure S1. Crystal structure of 3a-paco (with a chloroform solvent molecule). 

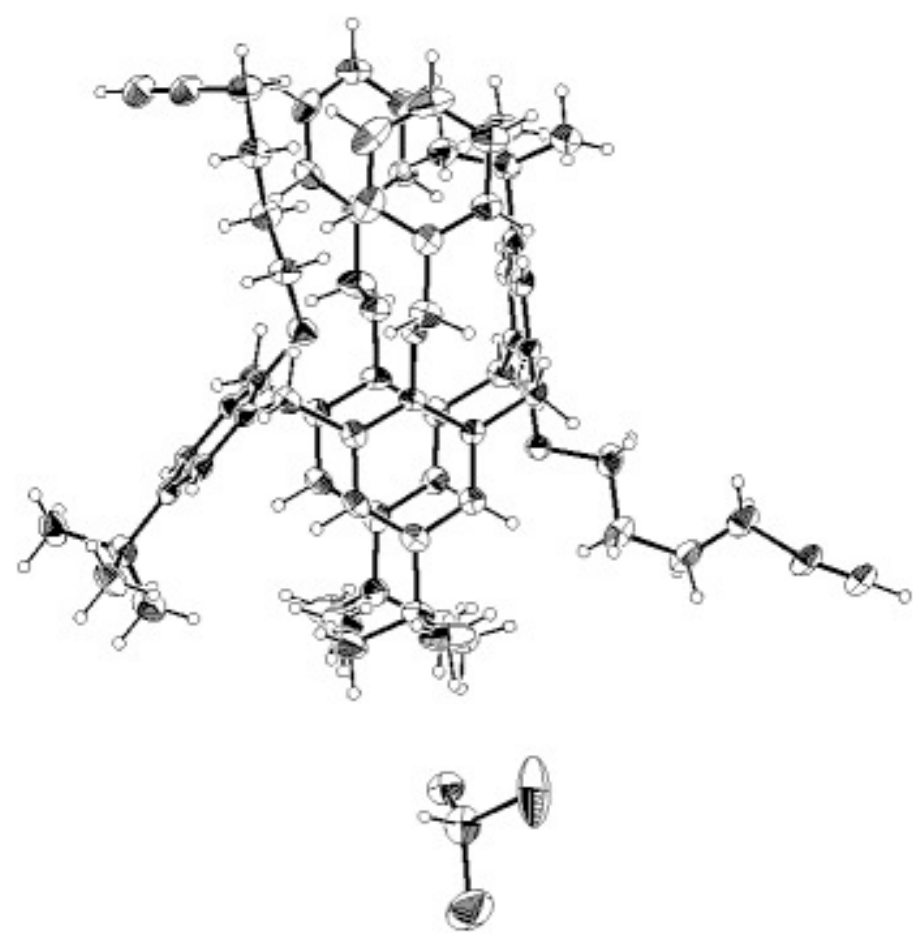

Table S3. Selected crystal data for 4a-paco.

Empirical formula

Formula weight

Temperature

Wavelength

Crystal system

Space group

Unit cell dimensions

Volume

Z

Density (calculated)

Absorption coefficient

$\mathrm{F}(000)$

Crystal size

Theta range for data collection
$\mathrm{C}_{64} \mathrm{H}_{72} \mathrm{O}_{4}$

905.22

$100(2) \mathrm{K}$

$0.71073 \AA$

Triclinic

P-1

$\mathrm{a}=10.0557(5) \AA$

$\alpha=74.269(2)^{\circ}$.

$\mathrm{b}=14.9830(8) \AA$

$\beta=76.871(2)^{\circ}$.

$\mathrm{c}=19.4748(11) \AA$

$\gamma=71.701(2)^{\circ}$.
2648.9(2) $\AA^{3}$

2

$1.135 \mathrm{Mg} / \mathrm{m}^{3}$

$0.069 \mathrm{~mm}^{-1}$

976

$0.35 \times 0.20 \times 0.20 \mathrm{~mm}^{3}$

2.01 to $29.57^{\circ}$. 
Index ranges

Reflections collected

Independent reflections

Completeness to theta $=29.57^{\circ}$

Absorption correction

Max. and min. transmission

Refinement method

Data / restraints / parameters

Goodness-of-fit on $\mathrm{F}^{2}$

Final $\mathrm{R}$ indices $[\mathrm{I}>2 \operatorname{sigma}(\mathrm{I})]$

$\mathrm{R}$ indices (all data)

Largest diff. peak and hole $-13<=\mathrm{h}<=13,-20<=\mathrm{k}<=20,-27<=1<=26$

59479

$14804[\mathrm{R}($ int $)=0.0252]$

$99.7 \%$

Semi-empirical from equivalents

0.9864 and 0.9763

Full-matrix least-squares on $\mathrm{F}^{2}$

14804 / 0 / 625

1.029

$\mathrm{R} 1=0.0491, \mathrm{wR} 2=0.1271$

$\mathrm{R} 1=0.0583, \mathrm{wR} 2=0.1361$

0.538 and -0.210 e. $\AA^{-3}$

Table S4. Selected crystal data for 3a-cone.

\begin{tabular}{|c|c|c|}
\hline Empirical formula & \multicolumn{2}{|l|}{$\mathrm{C}_{71} \mathrm{H}_{85} \mathrm{Cl}_{3} \mathrm{O}_{4}$} \\
\hline Formula weight & \multicolumn{2}{|l|}{1108.74} \\
\hline Temperature & \multicolumn{2}{|l|}{$100(2) \mathrm{K}$} \\
\hline Wavelength & \multicolumn{2}{|l|}{$0.71073 \AA$} \\
\hline Crystal system & \multicolumn{2}{|l|}{ Orthorhombic } \\
\hline Space group & \multicolumn{2}{|l|}{ Pbca } \\
\hline \multirow[t]{3}{*}{ Unit cell dimensions } & $\mathrm{a}=16.6918(8) \AA$ & $\alpha=90^{\circ}$ \\
\hline & $\mathrm{b}=20.6408(10) \AA$ & $\beta=90^{\circ}$ \\
\hline & $\mathrm{c}=37.0204(14) \AA$ & $\gamma=90^{\circ}$ \\
\hline Volume & \multicolumn{2}{|l|}{$12754.7(10) \AA^{3}$} \\
\hline $\mathrm{Z}$ & \multicolumn{2}{|l|}{8} \\
\hline Density (calculated) & \multicolumn{2}{|l|}{$1.155 \mathrm{Mg} / \mathrm{m}^{3}$} \\
\hline Absorption coefficient & \multicolumn{2}{|l|}{$0.190 \mathrm{~mm}^{-1}$} \\
\hline $\mathrm{F}(000)$ & \multicolumn{2}{|l|}{4752} \\
\hline Crystal size & \multicolumn{2}{|c|}{$0.28 \times 0.25 \times 0.18 \mathrm{~mm}^{3}$} \\
\hline Theta range for data collection & \multicolumn{2}{|l|}{1.64 to $29.35^{\circ}$} \\
\hline Index ranges & \multicolumn{2}{|c|}{$-23<=\mathrm{h}<=23,-28<=\mathrm{k}<=28,-51<=1<=51$} \\
\hline Reflections collected & \multicolumn{2}{|l|}{266215} \\
\hline Independent reflections & \multicolumn{2}{|c|}{$17509[\mathrm{R}(\mathrm{int})=0.0588]$} \\
\hline Completeness to theta $=29.35^{\circ}$ & \multicolumn{2}{|l|}{$100.0 \%$} \\
\hline
\end{tabular}


Absorption correction

Max. and min. transmission

Refinement method

Data / restraints / parameters

Goodness-of-fit on $\mathrm{F}^{2}$

Final $\mathrm{R}$ indices $[\mathrm{I}>2 \operatorname{sigma}(\mathrm{I})]$

$\mathrm{R}$ indices (all data)

Largest diff. peak and hole
Semi-empirical from equivalents

0.9666 and 0.9487

Full-matrix least-squares on $\mathrm{F}^{2}$

$17509 / 0 / 715$

1.071

$\mathrm{R} 1=0.0560, \mathrm{wR} 2=0.1379$

$\mathrm{R} 1=0.0757, \mathrm{wR} 2=0.1519$

0.668 and -0.621 e. $\AA^{-3}$

Table S5. Selected crystal data for 3a-paco.

Empirical formula
Formula weight
Temperature
Wavelength
Crystal system
Space group
Unit cell dimensions

Volume

Z

Density (calculated)

Absorption coefficient

$\mathrm{F}(000)$

Crystal size

Theta range for data collection

Index ranges

Reflections collected

Independent reflections

Completeness to theta $=21.97^{\circ}$

Absorption correction

Max. and min. transmission

Refinement method

Data / restraints / parameters
$\mathrm{C}_{70} \mathrm{H}_{84} \mathrm{O}_{4}+0.5 \mathrm{CHCl}_{3}$

1049.06

100(2) K

$0.71073 \AA$

Monoclinic

$\mathrm{P} 2(1) / \mathrm{c}$

$\mathrm{a}=14.4110(12) \AA$ $\alpha=90^{\circ}$.

$\mathrm{b}=20.9556(17) \AA$ $\beta=102.285(3)^{\circ}$.

$\mathrm{c}=20.7622(17) \AA$ $\gamma=90^{\circ}$.

6126.4(9) $\AA^{3}$

4

$1.137 \mathrm{Mg} / \mathrm{m}^{3}$

$0.131 \mathrm{~mm}^{-1}$

2260

$0.30 \times 0.10 \times 0.07 \mathrm{~mm}^{3}$

1.85 to $21.97^{\circ}$.

$-15<=\mathrm{h}<=14,0<=\mathrm{k}<=22,0<=1<=21$

68687

$7469[\mathrm{R}($ int $)=0.0687]$

$99.9 \%$

Semi-empirical from equivalents

0.9909 and 0.9617

Full-matrix least-squares on $\mathrm{F}^{2}$

7469 / 1116 / 786 
Goodness-of-fit on $\mathrm{F}^{2}$

Final R indices [I $>2 \operatorname{sigma}(\mathrm{I})]$

$\mathrm{R}$ indices (all data)

Largest diff. peak and hole
1.215

$$
\begin{aligned}
& \mathrm{R} 1=0.0868, \mathrm{wR} 2=0.1940 \\
& \mathrm{R} 1=0.0996, \mathrm{wR} 2=0.2000 \\
& 0.407 \text { and }-0.433 \mathrm{e} . \AA^{-3}
\end{aligned}
$$

\section{References and Notes}

${ }^{1}$ (a) Gutsche, C. D.; Iqbal, M. Org. Synth. 1990, 68, 234. (b) Gutsche, C. D.; Levine, J. A. J. Am. Chem. Soc. 1990, $55,5639$.

${ }^{2}$ Dijkstra, P. J.; Brunink, J. A. J.; Bugge, K. E.; Reinhoudt, D. N.; Harkema, S.; Ungaro, R.; Ugozzoli, F.; Ghidini, E. J. Am. Chem. Soc. 1989, 111, 7567.

${ }^{3}{ }^{13} \mathrm{C}$ NMR spectrum of $\mathbf{3 b}$ is made up of broad peaks.

${ }^{4}{ }^{13} \mathrm{C}$ NMR spectrum of $\mathbf{4 b}$ is made up of broad peaks.

5 The slight "decrease" of molecular weight may be due to the less rigid backbone of reduced polymer.

${ }^{6}$ The reaction condition was not optimized. Higher temperature was required to remove $\mathrm{Bn}$ groups but also degraded the polymer. 